\title{
STATUS OF SOLAR GENERATOR RELATED TECHNOLOGY DEVELOPMENT ACTIVITIES SUPPORTING THE JUICE MISSION
}

\author{
Carsten Baur ${ }^{(1)}$, Victor Khorenko ${ }^{(2)}$, Gerald Siefer $^{(3)}$, Virginie Inguimbert ${ }^{(4)}$, Seonyong Park $^{(5)}$, Bruno Boizot ${ }^{(5)}$, \\ Jacques C. Bourgoin $^{(5)}$, Mariacristina Casale ${ }^{(6)}$, Roberta Campesato ${ }^{(6)}$, Hans-Georg Schnell ${ }^{(7)}$, Andreas \\ Gerhard $^{(7)}$, Pietro Zanella ${ }^{(8)}$, Emanuele Ferrando ${ }^{(8)}$, Xavier Reutenauer ${ }^{(9)}$, Ed Bongers ${ }^{(10)}$, Ana Gras ${ }^{(11)}$ \\ (1) European Space Agency, Keplerlaan 1, 2201 AZ Noordwijk(The Netherlands),Email: carsten.baur@esa.int \\ (2) AZUR SPACE Solar Power GmbH, Theresienstr.2, 74072 Heilbronn (Germany), Email: \\ victor.khorenko@azurspace.com \\ (3) Fraunhofer Institute for Solar Energy Systems, 79110 Freiburg (Germany),Email: gerald.siefer@ise.fraunhofer.de \\ (4) ONERA/DESP, 2 avenue Edouard Belin, 31055 Toulouse Cédex 4 (France),Email: virginie.inguimbert@onera.fr \\ ${ }^{(5)}$ Laboratoire des Solides Irradiés, CNRS-UMR 7642, CEA-DRF-IRAMIS, Ecole Polytechnique, Université Paris- \\ Saclay, 91128 Palaiseau Cedex (France),Email: bruno.boizot@polytechnique.edu \\ (6) CESI S.p.A., Via Rubattino, 54, 20134 Milan (Italy),Email: mariacristina.casale@cesi.it \\ (7) Airbus DS GmbH, Ludwig-Bölkow-Allee, 81663 Munich (Germany), Email: hans- \\ georg.schnell@airbus.com \\ ${ }^{(8)}$ Leonardo - Finmeccanica, Viale Europa, 20014 Nerviano (Mi) (Italy), Email: \\ emanuele.ferrando@leonardocompany.com \\ (9) Thales Alenia Space France, 26 avenue J.F. Champollion, 31037 Toulouse Cedex 1 (France), Email: \\ xavier.reutenauer@thalesaleniaspace.com \\ (10) Airbus DS NL, Mendelweg 30,2333 CS Leiden (The Netherlands),Email:E.Bongers@airbusDS.nl \\ (11) INTA (SPASOLAB), Ctra. Ajalvir km.4, 28850 Torrejon de Ardoz (Madrid) (Spain), Email: grassa@inta.es
}

\begin{abstract}
The paper provides an overview of the current status of several technical development activities initiated by the European Space Agency (ESA) to support the JUICE mission to the Jovian system. First of all, the qualification status of the solar cells to be used in the JUICE mission will be reported. Then, the conclusions from a dedicated activity aiming at assessing the potential degradation of triple-junction solar cells upon primary discharges will be discussed. Finally, the results on the coupon tests currently running at ESA will be presented. The coupons consist of representative solar cell assemblies including coverglasses with a conductive Indium Tin Oxide (ITO) layer. Dedicated coverglass grounding technologies are tested on the coupons which connect the conductive coverglass surfaces to the panel ground. It will be shown how the resistivity of the materials used in the coupons evolves upon submission to extreme thermal cycles.
\end{abstract}

\section{INTRODUCTION}

The European JUpiter ICy moon Explorer (JUICE) mission to the Jovian system poses major challenges to the solar generator subsystem. Since the satellite on its cruise to Jupiter will make use of a Venus gravity assist, the satellite will be exposed to both, hot temperatures and high sun illumination and very cold temperatures and low sun illumination. Temperatures will thus range, between $+140-150^{\circ} \mathrm{C}$ for closest sun approach and extremely cold temperatures of $<-220^{\circ} \mathrm{C}$ reached around Jupiter when the satellite is in eclipse. Sun intensities received by the solar array are ranging between 2.4 suns (around $3300 \mathrm{~W} / \mathrm{m}^{2}$ ) and very low light intensities of down to $3.7 \%$ air mass "zero" (AM0) which are in the order of $50 \mathrm{~W} / \mathrm{m}^{2}$. Finally, the JUICE mission is also characterized by an extremely harsh irradiation environment (in terms of high energetic charged particles such as electrons and protons) which leads to high degradation on solar cell level due to particle irradiation. But also other effects like detrimental primary discharges as a consequence of high surface charging have to be taken into account. Dedicated technology development activities (TDAs) have been initiated by ESA in order to demonstrate the capability of a certain technology to sustain those extreme conditions. In the solar generator domain three activities are currently still ongoing or have been just finished:

1. JUICE Pre-qualification of Integrated LILT solar cells

2. Assessment of performance degradation of solar cells during the JUICE mission due to primary discharges

3. JUICE Coupon Test

This paper will give an overview on the status and/or the conclusions of those three activities. 


\section{ACTIVITY DESCRIPTIONS}

\subsection{JUICE Pre-qualification of Integrated LILT solar cells}

The objective of this activity was to run a full qualification test program in line with ECSS-E-ST-2008 [1] on bare cell and solar cell assembly (SCA) level, with a reduced number of test samples per subgroup, but including additional cell variants as a backup in the test program. In total 5 different solar cell variants have been produced by AZUR SPACE Solar Power GmbH (AZUR) including thin configurations $(80 \mu \mathrm{m})$ and configurations of standard thickness $(140 \mu \mathrm{m})$. An overview of the cell variants is given in Tab. 1. The difference in cell design ("Standard" vs. "LILT") is mainly related to a modification of the front side metallisation aiming at mitigating the so-called "flat spot effect"'[2-4]. However, as it was demonstrated in a precursor activity also the cells with standard design did not show any flat spot effect [5]. Thus, initially, Cell A with standard cell design and a thickness of $80 \mu \mathrm{m}$ was baselined for JUICE. The other combinations served as backup. A thin cell is preferred due to the mass criticality of the JUICE mission.

Table 1. Solar cell variants produced by AZUR.

\begin{tabular}{ccc}
\hline $\begin{array}{c}\text { Cell } \\
\text { structure }\end{array}$ & Cell Design & $\begin{array}{c}\text { Ge substrate } \\
\text { thickness }\end{array}$ \\
\hline A & Standard & $80 \mu \mathrm{m}$ \\
A & Standard & $140 \mu \mathrm{m}$ \\
A & LILT & $80 \mu \mathrm{m}$ \\
A & LILT & $140 \mu \mathrm{m}$ \\
B & Standard & $80 \mu \mathrm{m}$ \\
\hline
\end{tabular}

SCAs of all 5 cell variants have been manufactured by Airbus Defence and Space GmbH (ADS) and Leonardo Company (Leonardo). All cell and SCA variants have been submitted as a minimum to a screening test as described in [5] and a full beginning of life (BOL) characterisation covering illumination and temperature conditions around Venus and Jupiter. The screening of the cells and SCAs is required to sort out shunted cells which will clearly underperform under low intensity conditions.

A significant part of this activity was also dedicated to a quantification of the annealing effects that have been observed in precursor activities [5].

\subsection{Assessment of performance degradation of solar cells during the JUICE mission due to primary discharges}

The purpose of this activity was to test the vulnerability of the SCAs against potential degradation as a consequence of primary discharges when a nonconductive coverglass is used. The operation of solar cells under low intensity low temperature (LILT) conditions implies that shunt resistances created by primary discharges have a much stronger impact on the solar cell performance compared to missions where the cells are operated at higher sun intensities. Therefore, a total number of 20 samples have been submitted to 100 primary discharges each, in order to identify whether the cell performance would be affected and if "yes" to quantify a degradation factor.

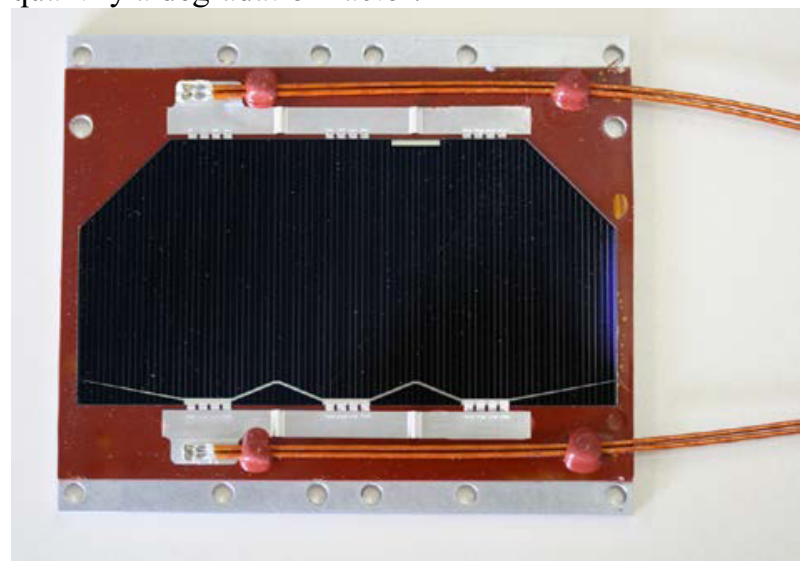

Figure 1. Solar cell "coupons" submitted to primary discharge tests. The coupon consisted of SCAs that were mounted on an Al plate with Kapton.

The samples that were tested were single-cell coupons as shown in Fig. 1. These "coupons" are SCAs mounted on Al plates covered with Kapton. This configuration was chosen in order to allow for an easier beginning of test (BOT) and end of test (EOT) characterisation that was performed at the Fraunhofer ISE. Since the BOT and EOT characterisation had to be performed also at LILT conditions the samples had to be mounted inside a cryostat. The Al plate with holes at the edges allowed for screwing the samples to the holder which was needed for arriving at a good thermal contact.

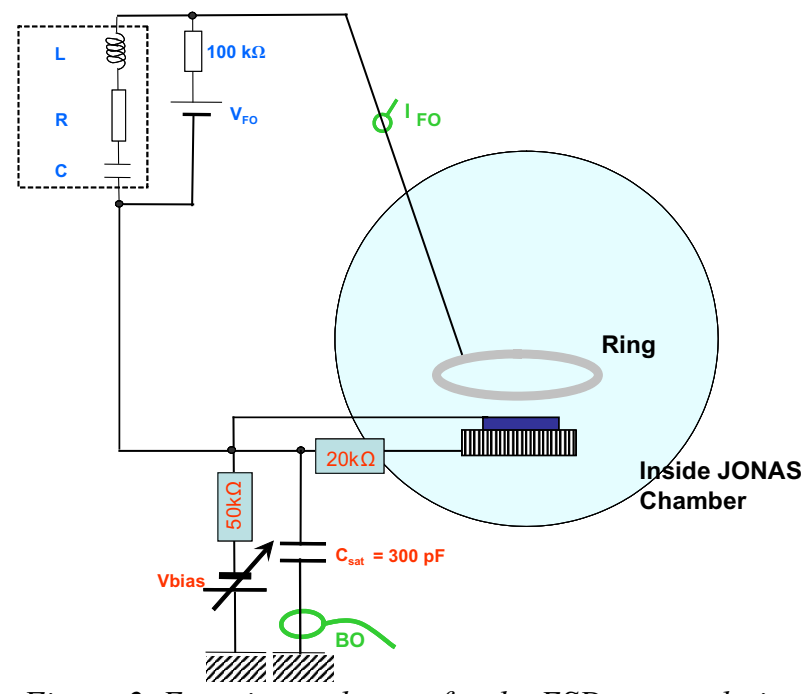

Figure 2. Experimental setup for the ESD accumulation test.

The electrostatic discharge tests have been carried out at 
ONERA at their JONAS facility using a flash-over simulator to represent the missing coverglasses on a real panel and assuming the discharge of a full panel as a worst case.

A schematic of the test setup is shown in Fig. 2 and is described in more detail in [6].

\subsection{JUICE coupon test}

The extreme thermal environment of the JUICE mission required a dedicated test program that aimed at demonstrating that existing substrate and PVA (photovoltaic assembly) technology could be used. All European solar generator and PVA suppliers were contacted to provide dedicated samples to be tested in the Mechanical Systems Laboratory (MSL) of ESAESTEC to be submitted to the test conditions and the test sequence as provided in Tab. 2 .

Table 2. Thermal cycling test sequence for JUICE solar array qualification, substrate samples and full coupons.

\begin{tabular}{lll}
\hline Step & No. of cycles & Conditions \\
\hline 1a & 2 thermal cycles & $+160^{\circ} \mathrm{C}$ to $-140^{\circ} \mathrm{C}$ \\
1b & 2 thermal cycles & $+80^{\circ} \mathrm{C}$ to $-160^{\circ} \mathrm{C}$ \\
$\mathbf{2}$ & $6 \mathrm{TV}$ cycles & $-100^{\circ} \mathrm{C}$ to $-240^{\circ} \mathrm{C}$ \\
3a & 3 thermal cycle & $+160^{\circ} \mathrm{C}$ to $-140^{\circ} \mathrm{C}$ \\
$\mathbf{3 b}$ & 3 thermal cycle & $+80^{\circ} \mathrm{C}$ to $-160^{\circ} \mathrm{C}$ \\
$\mathbf{4}$ & $90 \mathrm{TV}$ cycles & $-100^{\circ} \mathrm{C}$ to $-240^{\circ} \mathrm{C}$ \\
$\mathbf{5}$ & $45 \mathrm{TV}$ cycles & $-100^{\circ} \mathrm{C}$ to $-210^{\circ} \mathrm{C}$ \\
\hline
\end{tabular}

An overview of the tested samples are given in Tab. 3 .

Table 3. Overview of samples submitted to the thermal cycling test sequence in ESTEC facilities.

No Sample description

\begin{tabular}{l|l|}
\hline $\mathbf{1}$ & Kapton peel test samples \\
\cline { 2 - 2 } $\mathbf{2}$ & Flatwise tensile test samples \\
\cline { 2 - 2 } $\mathbf{3}$ & Full coupon with photovoltaic assembly (PVA) \\
\hline
\end{tabular}

\section{RESULTS}

\subsection{JUICE Pre-qualification of Integrated LILT solar cells}

The first major achievement in the framework of the pre-qualification activity was the identification of the solar cell baseline. BOL measurements revealed that cell A (cp. Tab. 1) suffers from a feature known as "majority carrier barrier effect" [7,8]. This effect is a fully reversible drop in cell performance, once the solar cell is operated below a certain threshold temperature. Fig. 3 shows the light I-V characteristic of two solar cells under LILT conditions. At $-120^{\circ} \mathrm{C}$ both cells provide almost identical power. However, once the cells are measured at $-150^{\circ} \mathrm{C}$, cell B clearly outperforms cell A. Cell A suffers from the majority carrier barrier effect which is obvious from the characteristic signature close to the open circuit voltage. It should be mentioned that this is true for all 4 variants of Cell A given in Tab. 1.
Since during the JUICE mission operating temperatures are dropping also below $-120^{\circ} \mathrm{C}$, it was decided to change to Cell B for the JUICE mission in an $80 \mu \mathrm{m}$ thick configuration as a baseline.

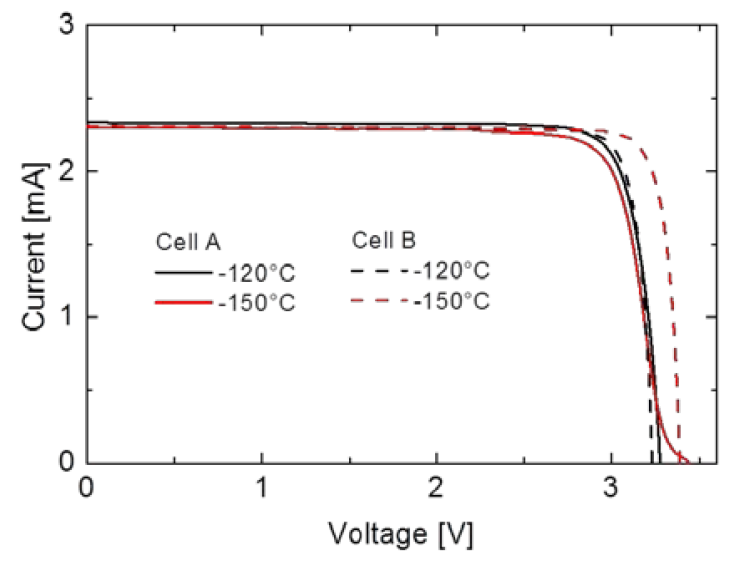

Figure 3. Light I-V curves of two solar cell variants measured under LILT conditions at $-120^{\circ} \mathrm{C}$ and $-150^{\circ} \mathrm{C}$.

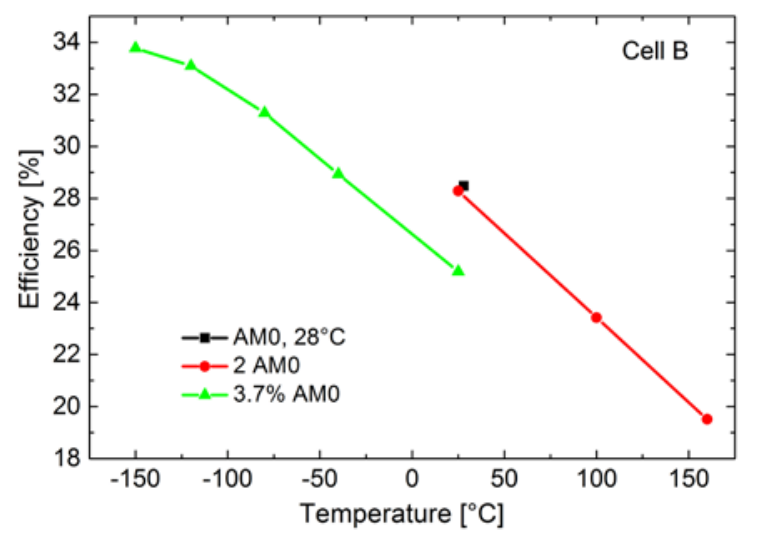

Figure 4. Evolution of the solar cell efficiency of cell B as a function of temperature for different illumination conditions.

Fig. 4 shows the evolution of the efficiency of cell B against temperature and for different illumination conditions. The black dot is the initial measurement under standard testing conditions (AM0, 28 ${ }^{\circ} \mathrm{C}$ ) performed at AZUR. The red points are derived from measurements performed at INTA Spasolab under high illumination conditions (up to $160^{\circ} \mathrm{C}, 2$ suns) to understand the performance of the solar cells during the Venus gravity assist. LILT characterisation of the cells were carried out at CESI (down to $-150^{\circ} \mathrm{C}, 3.7 \%$ AM0). For the high temperature part and the low temperature part the temperature coefficient follows nicely a straight line except for the very cold temperature regime where a saturation is reached. This is a known phenomenon and can be explained by tunnelling currents which at low temperatures start to become a dominant contributor [9]. Nevertheless, efficiencies at LILT conditions reach very high values of $34 \%$ (at $-150^{\circ} \mathrm{C}$, 
$3.7 \%$ AM0) with no sign of a flat spot effect.

Electron and proton irradiation tests have been performed at room temperature on cells and SCAs, respectively, with subsequent characterisation under LILT conditions. In addition, low temperature irradiation tests with in-situ LILT measurements have been performed by Ecole Polytechnique to assess the annealing factor that has been observed in precursor activities. The results of those tests are discussed in dedicated publications presented at this conference $[10,11]$. Other test activities such as UV, thermal cycling on cell and SCA level as well as the life test are not finished, yet.

\subsection{Assessment of performance degradation of solar cells during the JUICE mission due to primary discharges}

Bare cells of cell structure A and B of the two different thicknesses have been processed into SCAs and singlecell coupons (cp. Fig. 1) by ADS and Leonardo (applied coverglasses had no conductive layer). Tab. 4 lists the cell variants together with the number of samples per type that have been submitted to the ESD test. Numbers in brackets give the number of cells that showed degradation. Cell structure $\mathrm{A}^{*}$ is an $80 \mu \mathrm{m}$ cell where the same cutting process is applied as for the $140 \mu \mathrm{m}$ cell. The standard process for cutting $80 \mu \mathrm{m}$ cells differs slightly from the one applied for $140 \mu \mathrm{m}$ cells. Cell $\mathrm{A}^{*}$ was manufactured after the results of the first 10 samples were analysed, since it was initially assumed that the cutting process could have been responsible for the high number of degraded samples

Table 4. Coupon variants submitted to the ESD test. The number in brackets in the last column indicates the number of samples showing degradation.

\begin{tabular}{cccc}
$\begin{array}{c}\text { Cell } \\
\text { structure }\end{array}$ & $\begin{array}{c}\text { Thickness } \\
{[\boldsymbol{\mu m}]}\end{array}$ & $\begin{array}{c}\text { SCA } \\
\text { manufacturer }\end{array}$ & $\begin{array}{c}\text { No. of } \\
\text { samples }\end{array}$ \\
\hline A & 80 & ADS & $5(4)$ \\
A & 140 & Leonardo & $5(1)$ \\
A $^{*}$ & 80 & ADS & $1(1)$ \\
B & 80 & ADS & $2(0)$ \\
B & 140 & ADS & $2(0)$ \\
B & 80 & Leonardo & $2(0)$ \\
B & 140 & Leonardo & $3(2)$ \\
\hline
\end{tabular}

To track any potential degradation during the test sequence inside the test chamber, dark I-V measurements have been performed after the occurrence of every 10 ESD events (cp. Fig. 5). Furthermore, the location of the ESDs has been recorded by a video camera. Before starting the test and after the 100 ESDs electroluminescence, pictures were taken to detect potential shunts and their location (cp. Fig. 6). Fig. 7 shows the evolution of the dark current at a fixed voltage of $2 \mathrm{~V}$ as a function of the number of ESDs for all cells. When a degradation is observed (increasing dark current) this is mostly related to one single detrimental ESD event after which the dark current remains stable for the rest of the test program. Some cells also showed accumulative degradation as a consequence of multiple detrimental ESDs.

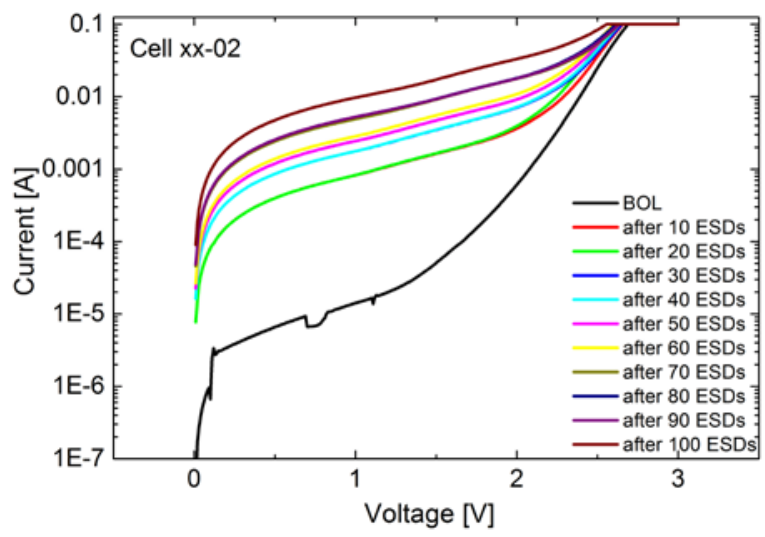

Figure 5. Dark current measurements of cell $x x$-02 after every 10 ESDs performed in situ at the JONAS facility of ONERA.

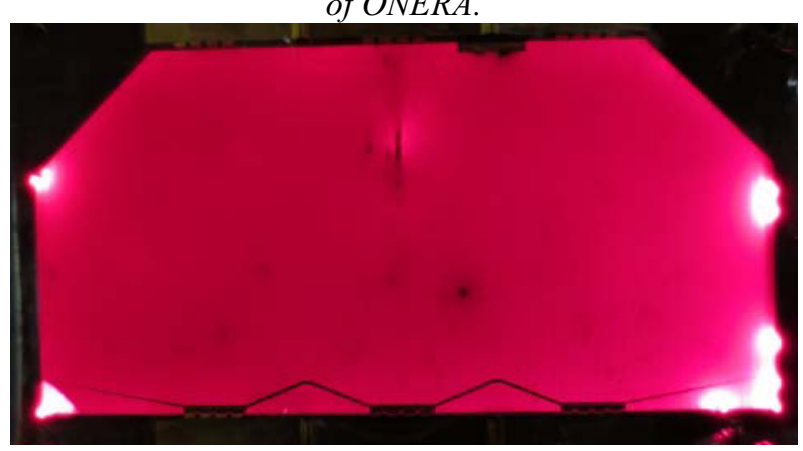

Figure 6. Electroluminescence picture of cell $x x-02$ after 100 ESDs. In this case, several ESD events have been introduced shunts (bright spots).

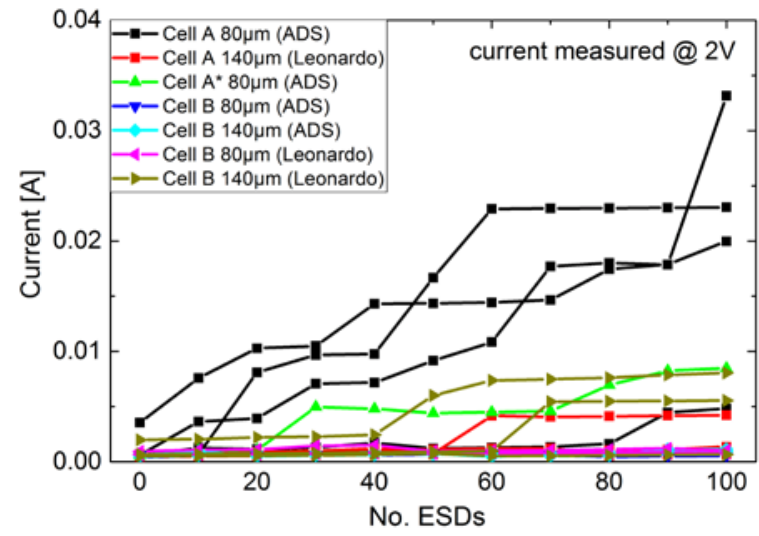

Figure 7. Dark current at a fixed voltage of $2 V$ as a function of number of ESDs.

Initially, only the first 10 coupons of Tab. 4 have been submitted to the ESD test campaign: while almost all the $80 \mu \mathrm{m}$ coupons by ADS showed some degradation, the opposite was true for the $140 \mu \mathrm{m}$ coupons from 
Leonardo where only one coupon showed some degradation. And this degradation could even not be correlated to an ESD event. Thus, the first assumption was that the thickness of the cell could be an important parameter for the vulnerability of the cell towards primary discharges. Since the cutting process of $80 \mu \mathrm{m}$ cells differs slightly from the $140 \mu \mathrm{m}$ cells, a dedicated $80 \mu \mathrm{m}$ cell was manufactured where the same cutting process as for the $140 \mu \mathrm{m}$ cell variant (cell $\mathrm{A}^{*}$ ) was applied. However, also this cell showed a clear degradation. Thus, this theory was discarded. In parallel, other coupons were manufactured using cell structure B (which had become the baseline for JUICE at that time). As can be seen in Fig. 7, in case of cell B only 2 out of 9 samples show some degradation and for 4 cells with $80 \mu \mathrm{m}$ thickness even no degradation is observed. However, since cell A and cell B only differ slightly in their epitaxial structure, the cell structure itself is not considered to play a role in this test. Thus, it was concluded that basically all cell variants can potentially be affected by primary discharges.

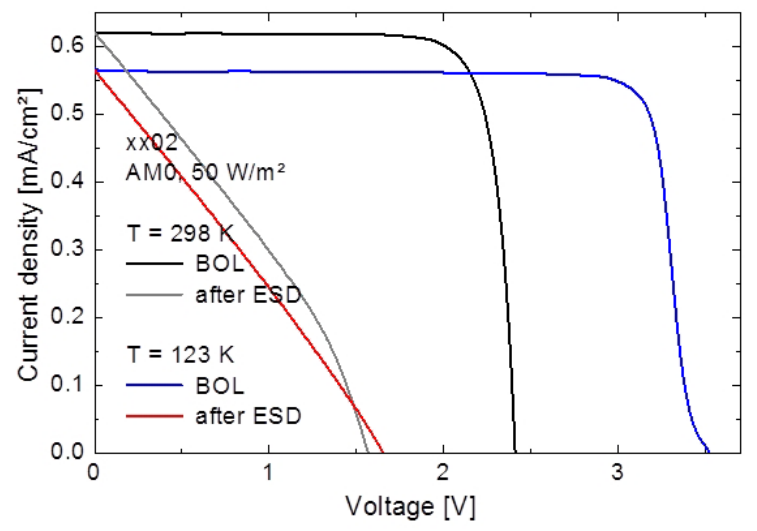

Figure 8. Light I-V curves of a coupon before and after ESD testing under low intensity conditions at room and low temperature.

The impact of the degradation was subsequently quantified by I-V measurements under LILT conditions at Fraunhofer ISE. An example of a coupon before and after the ESD test is shown in Fig. 8. There, light I-V measurements are shown under low intensity conditions at room temperature and $-150^{\circ} \mathrm{C}$. As mentioned before, the cumulative ESD events introduced one or more shunts at the cell edges leading to a significant drop in cell performance under those conditions. It should be highlighted that under full AM0 this drop in cell performance is much less. While under LILT conditions the drop in efficiency is almost $85 \%$, the same cell shown in Fig. 8 would only drop by $10 \%$ under AM0 (room temperature) conditions.

However, as a conclusion of these results, it is of major importance to protect the solar cells against primary discharges for the JUICE mission. Due to the fact that the solar generator is requested to meet a very stringent equipotential requirement of $1 \mathrm{~V}$, the coverglasses are anyway covered with a conductive Indium Tin Oxide (ITO) layer. This together with an appropriate coverglass grounding network should protect the solar cells against primary discharge events to happen.

\subsection{JUICE coupon test}

As explained in 2.3 all European solar generator and PVA suppliers were invited to provide their existing technology for tests under JUICE representative conditions. In a first step, only Kapton peel and flatwise tensile test samples were submitted to the test sequence. Samples were provided by Thales Alenia Space (TAS) (with Patria), Airbus Defence and Space NL (ARA Mk4 samples from Airborne Composites and ATK) as well as by ADS (Eurostar 3000 substrate). All samples have been successfully submitted to the thermal cycling test sequence without significant drop of bond strength when compared to uncycled reference samples. Subsequently, two test campaigns with full coupons (test article No. 3 in Tab. 2) were launched which have just recently been finished. In total, 4 coupons have been tested: 2 front side and 2 back side coupons. The separation into back and front side coupon was necessary to allow for a good thermal contact with the "cryo box" side wall that was used in the test facility where the cryogenic cycles were performed (and thus a significant reduction of test time). The two front side coupons are shown in Fig. 5. The dimension of the coupons was in all cases $410 \times 260 \mathrm{~mm}^{2}$ due to the limitations of the cryo box. Leonardo laid down 2 strings of 8 and 16 cells in series, respectively, on a substrate provided by TAS (manufactured by Patria). ADS did 4 strings of 5 cells in series on a Eurostar 3000 substrate. Both substrate technologies have significant space heritage of several ESA and commercial missions. One back side coupon per company was equipped with the typical back side elements such as blocking diodes, bleeder resistors and thermal sensors. The substrate used by Leonardo for the back side coupon was again provided by TAS/Patria while the substrate for the back side coupon from ADS was of Ara Mk4 type provided by ADS NL (Airborne Composites). As explained above, there is a stringent requirement to meet an equipotential on the solar generator of $1 \mathrm{~V}$. This made the application of ITO coated coverglasses mandatory. Special attention was therefore paid on a possible degradation of the resistivity of the ITO layers and/or different coverglass grounding concepts applied on the test coupons.

In Fig. 9, it can be seen that both companies used patches to interconnect the conductive surfaces of the coverglasses. While Airbus DS GmbH was using one specific coverglass grounding technology, Leonardo Company decided to test several combinations of patches and adhesives. Also in one string, a solution was applied where the coverglasses were directly 
connected to the shunt diode. However, this solution has to be discarded for the JUICE mission since it does not allow to meet the $1 \mathrm{~V}$ equipotential requirement. On the contrary, each coverglass will have the potential of the cell it is connected to, leading to a quite inhomogeneous potential distribution.
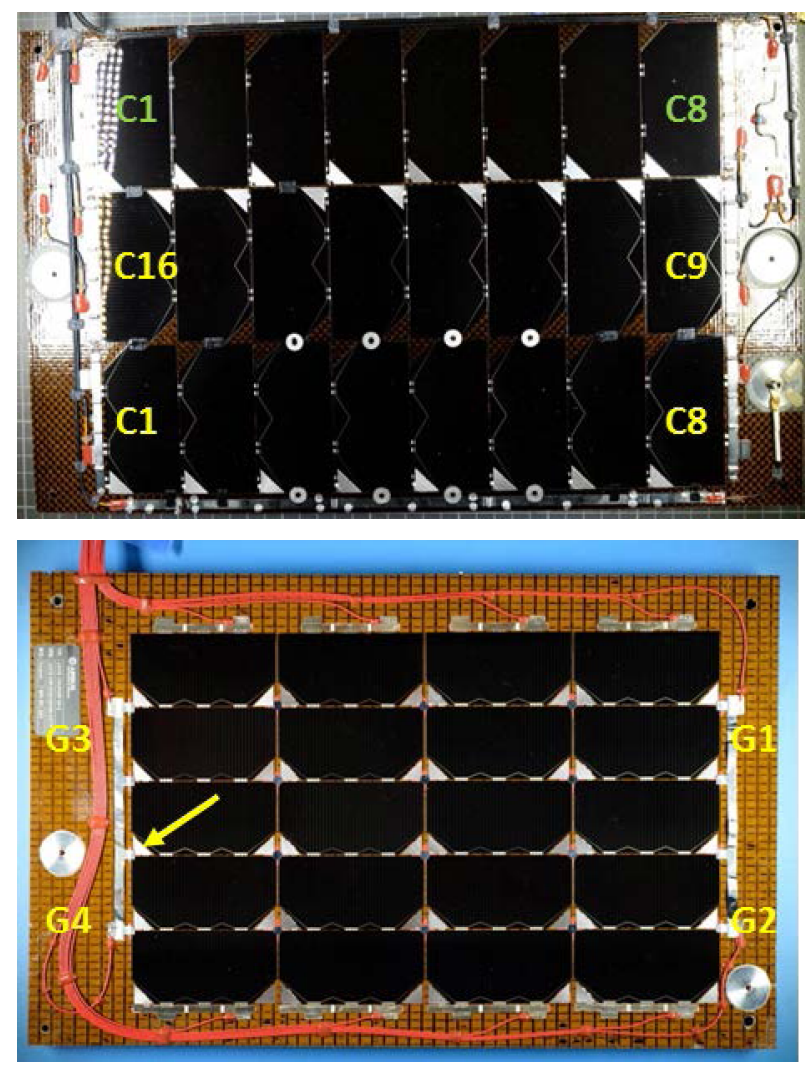

Figure 9. Front side coupons of Leonardo company (top) and ADS (bottom).

Before and after each test step (cp. Tab. 2), the coupons were investigated by visual inspection and an electrical health check was performed (including flasher test and electroluminescence measurements). During the cycling, the electrical elements were checked for continuity and an insulation check (cells vs. substrate) was performed during two cycles (beginning and end of each test sequence). Overall, the test demonstrates a significant confidence in most of the electrical elements. However, a few points of concern have been found which are currently subject to a more in-depth investigation:

1. On the Leonardo coupon, one string showed a slight degradation at $\mathrm{P}_{\max }$ of $4 \%$ after bake-out step $1 \mathrm{~b}$ of Tab. 2 which then remained stable during the test campaign. All other strings on the Leonardo and ADS coupons did not show any degradation at all. Therefore, it could be concluded that the solar cells do not suffer from the cycling profile.

2. A coverglass crack was noticed on one of the ADS
SCAs after step 4 which affected the coverglass grounding path (see below).

3. Some components on the back side coupons of both companies lost contact to the substrate (adhesive failure) without loss of functionality of the component itself.

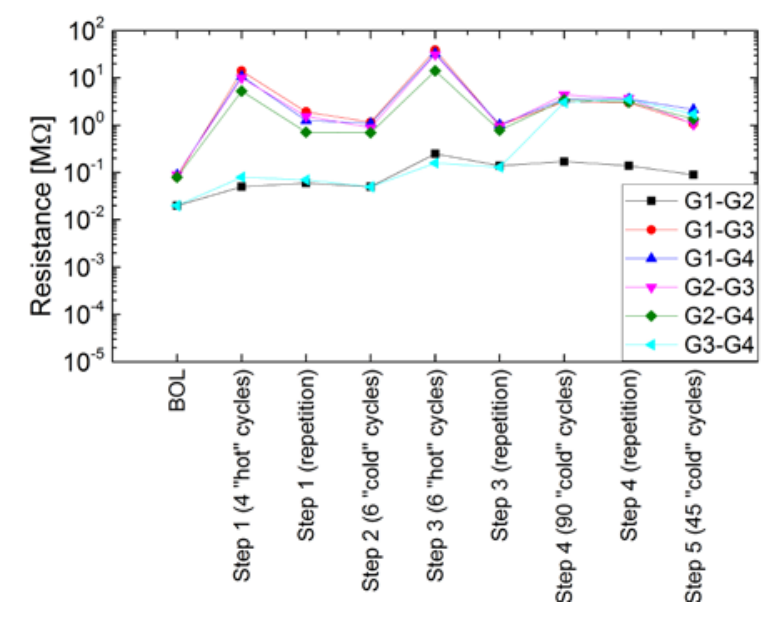

Figure 10. Resistance evolution of coverglass grounding network on ADS front side coupon.

Apart from those points, the major interest of the test campaign was focussed on the performance of the coverglass grounding network solutions. Fig. 10 shows the evolution of the coverglass grounding network resistivity as a function of the cycling steps. In order to have more information about the resistivity as a function of the current path length, the coverglass network grounding bars on each side of the coupon were cut in the middle and resistance measurements were performed between the 4 points G1 to G4 shown in Fig. 9 (bottom). The resistance was derived by applying $50 \mathrm{~V}$ between any two points and measuring the current. Obviously, the current paths with the shortest distance G1-G2 and G3-G4 - have the lowest resistivity. For those points mainly the resistivity of the ITO itself is measured. For all the other measurements, the resistivity values are quite similar which could be expected since the current has to pass over the same minimum number of patches (3). While before any thermal cycling the resistance was in all cases below $100 \mathrm{k} \Omega$, a strong increase was observed after the first step with values in the order of $10 \mathrm{M} \Omega$. However, repeating this measurement after a few days showed a significant recovery with values being now in the range of $1-2 \mathrm{M} \Omega$. The cold cycles in step 2 did not lead to any change in the resistivity. However, additional "hot" cycles in step 3 again lead to very high values which again recovered after a few days. The last cycles consisting of two sequences of "cold" cycles did not lead to a further increase of the resistance resulting in a final resistivity (for the long current paths) of 1-2 M $\Omega$. The sudden increase of the resistivity between G3-G4 can be 
explained by a crack in the coverglass of cell 3 in string 4 (numbering the strings from right to left in Fig. 9 bottom) exactly at the position of the grounding bar connection apparently interrupting the ITO coating (arrow in Fig 9 bottom). This results in a current path equivalent to the others (where the current has to flow over at least 3 patches). Overall, it can be concluded that the resistivity of the coverglass grounding network increased by roughly one order of magnitude with respect to the BOL measurement. However, the maximum values in terms of resistivity would still allow for meeting the $1 \mathrm{~V}$ equipotential requirement.

On the coupon from Leonardo, the interesting measurements with respect to the grounding network solutions were done on string 2 (consisting of 16 cells in series) since the solution used on string 1 (coverglasses connected to cell) cannot be used for the JUICE mission as explained above. Patches are applied between the short edges of adjacent cells and from the short edges to the grounding bar (only on one side). 3 different coverglass grounding solutions were applied labelled solution A, B and C. An overview of which coverglass grounding solution has been used between the different cells of string 2 of the Leonardo coupon is given in Tab. 4.

Table 4. Overview on which coverglass grounding solution has been used between the different cells of string 2 of the Leonardo coupon.

\begin{tabular}{ll}
\hline Cells in string 2 & CG network solution \\
\hline $\mathbf{1 , 1 6}$ & A \\
$\mathbf{2}, 15$ & A \\
$\mathbf{3}, 14$ & B \\
$\mathbf{4}, 13$ & B \\
$\mathbf{5 , 1 2}$ & C \\
$\mathbf{6 , 1 1}$ & C \\
$\mathbf{7 , 1 0}$ & A \\
$\mathbf{8 , 9}$ & A \\
\hline
\end{tabular}

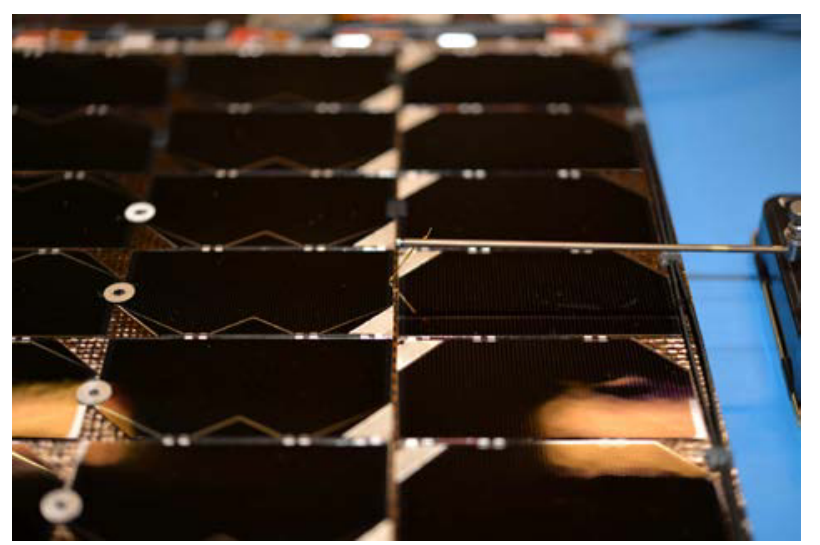

Figure 11. Application of a measurement probe on top of the outer edge of the coverglass. The other electrical connection is done via the grounding bar on the left side (not visible in this picture).
Measurements of the resistance was carried out by using on one side the connection of the grounding bar and by probing on the other side directly on top of the coverglass (see Fig. 11). By this means, the current had to flow over the length of two cells and over two grounding patches per cell pair (one patch between the cells +1 patch from cell to grounding bar). As for the ADS coupon, resistances were derived from applying $50 \mathrm{~V}$ between the two contact points and measuring the current.

The evolution of the resistance of the coverglass grounding network is given in Fig. 12 showing that all solutions exhibit a resistance of clearly less than $100 \mathrm{k} \Omega$ at the end of the test. For solutions A and B there is at least one path that shows a significant change after one of the test steps. Only solution $\mathrm{C}$ remains very stable. However, it has to be pointed out that the statistics are very poor.

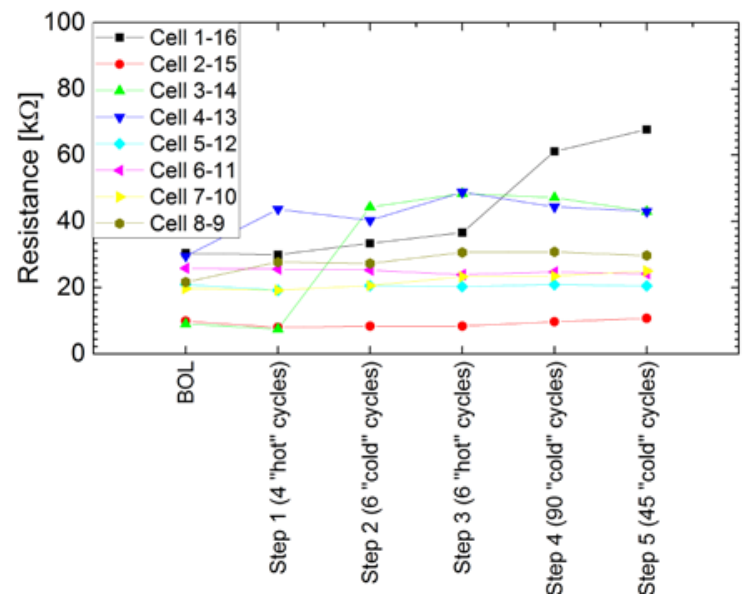

Figure 12. Resistance evolution of coverglass grounding network on Leonardo front side coupon.

Additional measurements have been performed probing each individual coverglass separately (on both sides a probe was placed directly onto the coverglass) in order to check the performance of the ITO layer itself. In average over the 24 cells the resistivity changed from $14.2 \mathrm{k} \Omega$ before the test to $11.5 \mathrm{k} \Omega$ after the test. These values are well in line with those quoted by Qioptiq in the order of $5 \mathrm{k} \Omega / \mathrm{sq}$. Taking into account that the way of measuring gives rise to some variability in the results it can be concluded that the ITO resistivity remains very stable during the cycling test.

Additional coupons of both companies are currently being manufactured and are planned to be tested in the upcoming months. On one side, these additional tests are aimed at improving the coverglass grounding network solutions taking into account what has been learned from the present tests and on the other side shall increase statistics. 


\section{CONCLUSION}

The status of running or finished TDAs initiated by ESA to support the JUICE mission has been provided. In the pre-qualification activity the major finding was the detection of the majority carrier barrier effect in one cell structure that led to a reorientation of the cell baseline. Test activities are ongoing with UV, thermal cycling and life test still to be performed. A significant amount of effort went into the electron and proton irradiation campaigns which are discussed in separate contributions to this conference $[10,11]$.

The activity related to the evaluation of potential performance losses upon primary discharges has been finished. It was concluded that there is a potential risk that one ESD event can cause an unacceptable degradation of the solar cell performance under LILT conditions. Therefore, a protection against primary discharges became mandatory for the JUICE mission. Since an ITO coating together with a coverglass grounding network was meanwhile already baselined due to the $1 \mathrm{~V}$ equipotential requirement on the solar array, this conclusion did not lead to a design change. It was mentioned that under AM0 conditions the observed degradations were much less compared to LILT conditions. However, the findings within this activity are concerning enough to justify the implementation of a dedicated activity to investigate further whether primary discharges could cause detrimental performance losses also for other more standard missions (operating under AM0 and above).

Finally, the coupon test campaign with 4 coupons (two front and two back side coupons) has been successfully completed with a few observations that still need to be investigated. However, it can be concluded that the PVA basically survives the JUICE thermal environment and that there are also coverglass grounding network solutions that survive the cycling profile and meet the stringent equipotential requirement.

\section{ACKNOWLEDGEMENT}

The authors would like to thank a number of people that contributed significantly to the generation of the huge amount of data that served as a basis for the analysis provided in this paper: Elvira Fehrenbacher, Michael Schachtner, Alexander Wekkeli from Fraunhofer ISE, Bernd Lehmann, Stephane Roure, Carl Hall, Bob Witteveen, Natascha Klein from ESA, Gerald Jüngst, Ainhoa Martínez de Olcoz, Alberto Vegas at INTA and Jean-Michel Siguier from ONERA. Furthermore, C. Baur likes to thank his colleagues from TEC-EPG: Emilio Fernandez, Paul Hodgetts, Antonio Caon, José Ramón González and Gianfelice D’Accolti for fruitful discussions on testing and interpretation of test results. Last but not least, C. Baur likes to thank the JUICE project team Sam Verstaen, Robert Furnell and Fabio Panin from ESA and Thomas Schirmann and Didier Loche from Airbus DS Toulouse for the good collaboration and support in the execution of the TDAs.

\section{REFERENCES}

[1] ECSS-E-ST-20-08C, Rev. 1, "Space engineering Photovoltaic assemblies and components." 2012.

[2] V. G. Weizer and J. D. Broder, "On the cause of the flat-spot phenomenon observed in silicon solar cells at low temperatures and low intensities," $J$. Appl. Phys., vol. 53, no. 8, pp. 5926-5930, 1982.

[3] C. J. Gelderloos, K. B. Miller, R. J. Walters, G. P. Summers, and S. R. Messenger, "Low intensity low temperature performance of advanced solar cells," in Proceedings of the 29th IEEE Photovoltaics Specialists Conference, New Orleans, Louisiana, USA, 2002, pp. 804-807.

[4] R. Campesato, C. Flores, G. Damonte, and S. Taylor, "Modelling and analysis of multi junction solar cells at low temperature/low intensity conditions," in Proceedings of the 22nd European Photovoltaic Solar Energy Conference, Milano, Italy, 2007, pp. 680-683.

[5] C. Baur, V. Khorenko, G. Siefer, J. C. Bourgoin, M. Casale, R. Campesato, S. Duzellier, and V. Inguimbert, "Development status of triple-junction solar cells optimized for low intensity low temperature applications," in 39th IEEE Photovoltaic Specialists Conference, 2013, pp. 3237-3242.

[6] V. Inguimbert, P. Sarrailh, J.-C. Mateo-Velez, J.M. Siguier, C. Baur, B. Boulanger, A. Gerhard, P. Pelissou, M. Sevoz, and D. Payan, "Measurements of the Flashover Expansion on a Real-Solar PanelPreliminary Results of EMAGS3 Project," IEEE Trans. Plasma Sci., vol. 41, no. 12, pp. 3370-3379, Dec. 2013.

[7] R. Hoheisel, R. J. Walters, and A. W. Bett, "Low temperature effects in photovoltaic devices for deep space missions," in Proceedings of the 42nd IEEE Photovoltaic Specialist Conference, 2015, pp. 1-5.

[8] R. Hoheisel and A. W. Bett, "Experimental Analysis of Majority Carrier Transport Processes at Heterointerfaces in Photovoltaic Devices," IEEE J. Photovolt., vol. 2, no. 3, pp. 398-402, Jul. 2012.

[9] J. A. Del Alamo and R. M. Swanson, "Forwardbias tunneling: a limitation to bipolar device scaling," IEEE Electron Device Lett., vol. EDL-7, no. 11, pp. 629-631, 1986.

[10] V. Khorenko, C. Baur, G. Siefer, M. Schachtner, S. Park, B. Boizot, J. C. Bourgoin, M. Casale, and R. Campesato, "BOL and EOL characterization of AZUR 3G LILT solar cells for ESA JUICE mission," at this conference.

[11] S. Park, J. C. Bourgoin, O. Cavani, V. Khorenko, C. Baur, and B. Boizot, "Origin of the degradation of triple junction solar cells at low temperature," at this conference. 